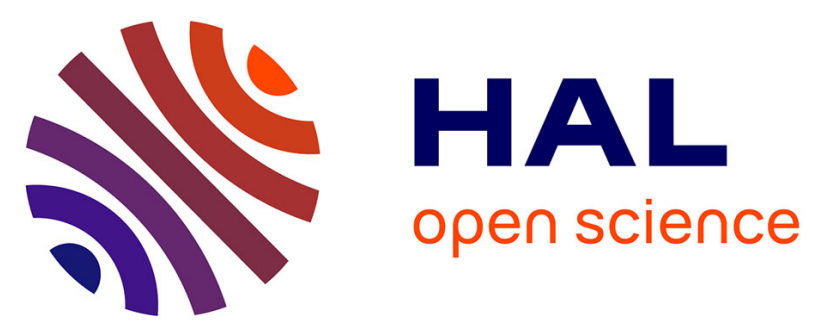

\title{
In situ and ex situ Investigations of Oxygen, Light and Temperature Influence on Halide Perovskites: Absorption and Transport Properties
}

Stefania Cacovich, Amelle Rebai, Hung-Ju Lin, Jean Rousset, Christophe Longeaud

\section{To cite this version:}

Stefania Cacovich, Amelle Rebai, Hung-Ju Lin, Jean Rousset, Christophe Longeaud. In situ and ex situ Investigations of Oxygen, Light and Temperature Influence on Halide Perovskites: Absorption and Transport Properties. IEEE 46th Photovoltaic Specialist Conference (PVSC 46), Jun 2019, Chicago, United States. hal-02280585

\section{HAL Id: hal-02280585 \\ https://hal-centralesupelec.archives-ouvertes.fr/hal-02280585}

Submitted on 20 Jul 2021

HAL is a multi-disciplinary open access archive for the deposit and dissemination of scientific research documents, whether they are published or not. The documents may come from teaching and research institutions in France or abroad, or from public or private research centers.
L'archive ouverte pluridisciplinaire HAL, est destinée au dépôt et à la diffusion de documents scientifiques de niveau recherche, publiés ou non, émanant des établissements d'enseignement et de recherche français ou étrangers, des laboratoires publics ou privés. 


\title{
In situ and ex situ investigations of oxygen, light and temperature influence on halide perovskites : absorption and transport properties
}

\author{
Stefania Cacovich ${ }^{1}$, Amelle Rebai ${ }^{1}$, Hung-Ju Lin ${ }^{1}$, Jean Rousset ${ }^{1,2}$ and Christophe Longeaud ${ }^{3,1}$ \\ ${ }^{1}$ IPVF, Ile-de-France Photovoltaic Institute (IPVF), 30 Route Départementale 128, Palaiseau, 91120, \\ Palaiseau, France \\ ${ }^{2}$ EDF R\&D, 30 Route Départementale 128, 91120, Palaiseau, France \\ ${ }^{3}$ GeePs, CNRS (UMR8507), Centrale-Supelec, Pierre and Marie Curie University, Paris-Sud 11 University, \\ 11 rue Joliot Curie, 91190, Gif sur Yvette, France
}

\begin{abstract}
In this work multi-cations hybrid perovskite thin film properties were explored by characterization techniques that proved to be very powerful for inorganic thin films. In particular, we report the evolution of the ambipolar diffusion length under different environmental or thermal stresses by Steady State Photocarrier Grating, directly observing the effects of oxygen absorption/desorption onto the material electronic properties. Moreover, the effects of illumination on the absorption properties were investigated via Fourier Transform Photocurrent Spectroscopy, a technique that allows to access to weak absorptions $(\mathrm{E}<\mathrm{Eg})$.
\end{abstract}

\section{INTRODUCTION}

Over the last years we have witnessed a tremendous interest into hybrid organic-inorganic perovskite solar cells (PSCs) research. Indeed, this emerging class of devices has become increasingly popular due to the opportunity of reaching a high-power conversion efficiency, while being compatible with wet chemistry processing for large area devices. The excellent photovoltaic performance of halide perovskites goes along with a high photoluminescence yield that makes them suitable for a wide range of photonic devices and various optoelectronic applications, such as photodetectors, lasers and light emitting diodes. The progress in PSCs originated from rapid advances in precursor formulation, fabrication methods and device architecture - however, most of the progress has been obtained through empirical device improvements, and several key questions still remain unanswered. Open issues include the optimal chemical composition of the perovskite films, ion migration, scalable fabrication routes, device architecture and stability in operation. Moreover, one of the main advantages of hybrid halide perovskite is the tunability of the crystal size and the ensuing band gap. The archetypal $\mathrm{CH}_{3} \mathrm{NH}_{3} \mathrm{PbI}_{3}$ is only one of the many possible hybrid perovskites composition. All the main components (i.e. organic molecule, metal and halides) can be substituted or mixed with different chemical species, resulting in complex or intermixed compounds. Due to the complexity of the system, often traditional characterization tools are not able to unveil the physical processes underlying the working principles of the solar cells. New approaches in the characterization of the materials are thus required to overcome these hurdles, leading to a deep understanding of the carrier transport and recombination processes. Moreover, temperature, light and air/moisture exposure were proven to be responsible for the drastic reduction in device performance. To overcome this limitation, it is thus crucial to understand the degradation pathways of the structures involved and their impact on the optoelectronic properties of the active layer.

In this communication we show that techniques applied successfully to the study of inorganic thin films can also bring some insight in the properties of perovskites thin films.

Depending on the measurement technique there are huge differences in reported charge carrier diffusion length values, varying from $100 \mathrm{~nm}$ and a few $\mu \mathrm{m}$ for thin films, and even exceeding tens or hundreds of microns for monocrystals. The optimal experimental conditions and methods to measure this crucial parameter are still under debate in the perovskite community. In this work, we have measured the electronic properties of different perovskite thin films by employing steady state photocarrier grating (SSPG) $[1,2]$ since this approach allows to directly and unambiguously measure the ambipolar diffusion length $\left(L_{d}\right)$ and the mobility $\times$ lifetime product $(\mu \tau)$ of the carriers. It is worth noting that in the literature these important material properties, mobility and lifetime, have been mostly estimated with experiments based on transient techniques. We have developed an automated, fast and ready-to-use bench coupled with a cryostat, allowing us to measure the ambipolar diffusion lengths of perovskite thin films under various vacuum and temperature conditions within a few minutes [3, 4]. One of the main advantages of this bench lies in the opportunity to rapidly and accurately determine the ambipolar diffusion length of the carriers. In addition, a complete knowledge of transport properties can be achieved by performing dark and steady state photocurrent measurements, leading to the estimation of the activation energy and the mobility $\times$ lifetime product $(\mu \tau)$ of the carriers (not shown here for the lack of space).

Fourier Transform Photocurrent Spectroscopy (FTPS) can be employed to obtain the absorption spectra of perovskite 
thin films $[5,6]$. This characterization method allows to have access to the weak absorption $\left(\mathrm{E}<\mathrm{E}_{\mathrm{g}}\right)$ region of the film, providing detailed insights on the photophysics of hybrid perovskites and eventually revealing the present of deep defect distributions in the gap of the material.

The potentialities of these techniques are exemplified in the following paragraph in which they are applied to different films of triple cations perovskites containing different ratios of $\mathrm{Cs}$ or $\mathrm{Rb}$ prepared at IPVF [7].

\section{EXPERIMENTAL RESULTS AND DISCUSSION}

In the following examples several perovskite compositions were tested and compared under different stresses conditions. In Fig. 1 the influence of the temperature on the ambipolar diffusion length is shown for a $\mathrm{CH}_{3} \mathrm{NH}_{3} \mathrm{~Pb}(\mathrm{I}-\mathrm{Rb})(\mathrm{MaFaRb})$ thin film. The sample was heated from room temperature $(\sim 295 \mathrm{~K})$ up to $360 \mathrm{~K}$ and the SSPG measurements were carried out every $20 \mathrm{~K}$ steps. Moreover, the sample was maintained at $360 \mathrm{~K}$ during $1 \mathrm{~h} 20$ to emphasize the influence of a temperature annealing. It can be seen in Fig. 1 that in the case of the MaFaRb compound, the diffusion length decreases since the first measurements, dropping down to very low values (Black squares). After a cooling down, a second heating treatment was applied to the sample up to $380 \mathrm{~K}$ (Red circles). As long as the MaFaRb compound is kept under vacuum, the temperature decrease does not allow to recover the initial values of the diffusion length that remained rather low as shown by a third increase of temperature (Blue stars).

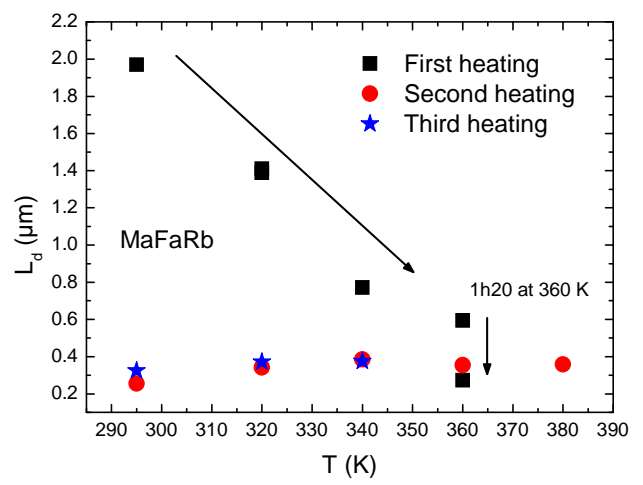

Fig 1. Effects of temperature on a MaFaRb perovskite thin film diffusion length, measured by the SSPG technique.

The same behavior was observed also on MaFaCs thin films. To discriminate from the influence of temperature and vacuum we have performed a measurement of $L_{d}$ on a $\mathrm{MaFaCs}$ film at room temperature but with or without the vacuum. After a steady low value of $L_{d}$ was obtained at $295 \mathrm{~K}$ the vacuum $\left(2 \times 10^{-6} \mathrm{mBarr}\right)$ was broken and $L_{d}$ measured regularly as function of time until it reaches some stabilization. As this point we started to pump again the cryostat to restore the vacuum situation and still measured regularly the evolution of $L_{d}$. The evolutions of $L_{d}$ during the vacuum $\mathrm{OFF}$ and vacuum $\mathrm{ON}$ situations are displayed in Fig. 2. One can see that it took almost 4 hours for $L_{d}$ to increase from $\sim 490 \mathrm{~nm}$ up to $\sim 1.1 \mu \mathrm{m}$ under air (vacuum OFF) and that $L_{d}$ decreased back to $\sim 490 \mathrm{~nm}$ once the vacuum was turned on again underlying the influence of air on the transport properties of the film.

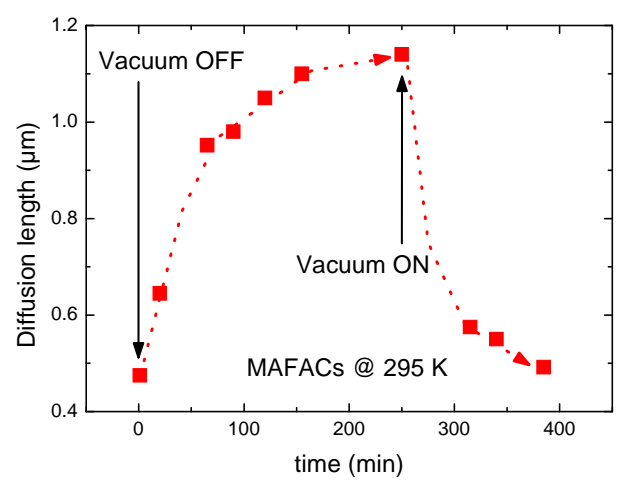

Fig 2. Effects of different environmental conditions on a MaFaCs perovskite thin film diffusion length, measured by SSPG technique.

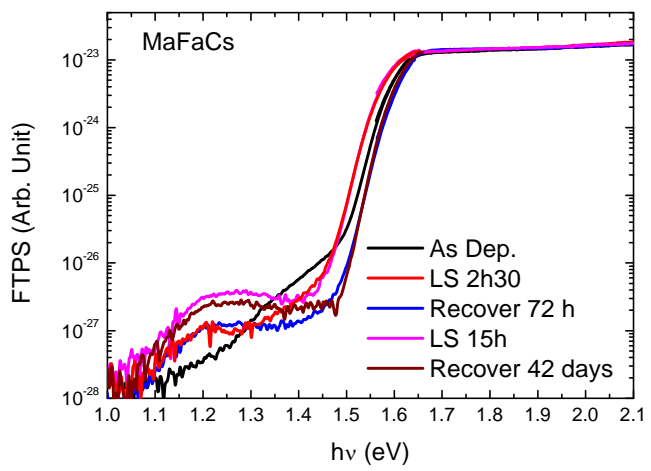

Fig 3. FTPS spectra for a triple cation perovskite thin film under different illumination conditions.

Finally, we investigated by means of FTPS the effects of continuous illumination, equivalent to $\mathrm{AM} 1.5 \mathrm{G}$, on a standard triple cation perovskite thin film (MaFaCs). In order to investigate the reversibility, or not, of light induced phenomena, we recorded different spectra : as-deposited, after $2 \mathrm{~h} 30$ of light soaking (LS), after $72 \mathrm{~h}$ of recovery under primary vacuum and dark, after $15 \mathrm{~h}$ of LS and after 42 days of recovery. These results are displayed in Fig. 3. The fast drop observed around $1.55 \mathrm{eV}$ corresponds to the onset of the band edge of the film. We can notice that in the as-deposited state a distribution of states gives rise to a contribution to the absorption around $1.4 \mathrm{eV}$. After $2 \mathrm{~h} 30$ of LS it can be seen that the band edge sets at a lower energy than in the as-deposited state and that a distribution of states was created around 1.25 $\mathrm{eV}$ whereas the contribution of the distribution of states at 1.4 
$\mathrm{eV}$ is weaker. After $72 \mathrm{~h}$ of recovery the band edge has shifted toward higher energies whereas the distribution of states around $1.25 \mathrm{eV}$ is almost unaffected and the distribution around $1.4 \mathrm{eV}$ has vanished. After $15 \mathrm{~h}$ of LS the band edge has moved toward low energies again and the distribution of deep states around $1.25 \mathrm{eV}$ has increased by a factor of 3 . A recovery of 42 days results in a blue shift of the band edge whereas the deep defect distribution remains quite as high.

Such behaviors have been observed on several films with different compositions.

\section{CONCLUSIONS}

We have illustrated the powerfulness of the SSPG, and FTPS techniques to investigate transport parameters and optical absorption of perovskite films, respectively.

SSPG appears as a very powerful tool to investigate on the ambipolar diffusion length of the films under study and its evolution with the different treatments that we have performed on the films. Experiments performed on different thin films revealed the influence of the temperature and air on the transport properties of perovskite thin films. The behaviors displayed in Figs. 1 and 2 have been explained in detail in a recent paper [7]. To summarize we believe that absorption of an air component (moisture or oxygen) of the air passivates a defect level and gives rise to rather high values of $L_{d}$. This defect level is activated by desorption of this air component during the study under vacuum of the electronic properties of the films, resulting in an enhancement of the recombination rate of both carriers. This desorption would be helped by the successive heating stages of the material, though it can be observed on some samples even without any heating. On the other hand, absorption of this component would passivate this defect level resulting in high ambipolar diffusion. Such a reversible behavior, improvement or degradation of the material properties, was also observed in photoluminescence and impedance studies by other groups that attributed this phenomenon to absorption/desorption of oxygen $[8,9,10]$. Therefore, we assume that the reversible improvement/ degradation of the transport properties of the studied films can be also attributed the interaction with oxygen.

Though the origin of this passivated/unpassivated defect level is not clearly identified, probably the presence of iodide vacancies, it would mean that oxygen is not always detrimental to the films properties and could be used as a treatment to be applied to devices to improve their performances before the deposition of the top electrode. Nevertheless, further work is needed to optimize the oxygenation of the material without irreversible degradations, and to encapsulate the device to avoid desorption of oxygen while the device is working under high illumination and high temperature when exposed to the sun.

The FTPS measurements have put into evidence the influence of a high illumination on the band edge and below gap absorption coefficient of the perovskite. The evolution of the absorption coefficient under LS is two-fold : a shrinking of the band gap and the creation of an absorption contribution around $1.25 \mathrm{eV}$, this latter being probably related to the creation of a defect states distribution. The amplitude of the defect density seems to depend on the duration of the lightsoaking process and insensitive to a recovery at room temperature, the sample being held under dark and under vacuum. On the other hand, the width of the band gap returns to its previous value after recovery, a behavior that indicates that these two phenomena, creation of a defect density and evolution of the band gap are not due to the same process since they have different kinetics.

In conclusion, SSPG and FTPS appear to be very powerful tools to investigate on the evolution of the opto-electronic properties of perovskite thin films under different conditions of stress (temperature, exposition to moisture and oxygen) and aging under high intensity light. However, in both cases more work is needed to link the observed behaviors and aging to structural or chemical evolution of the layers.

\section{REFERENCES}

[1] D. Ritter, E. Zeldov, K. Weiser, "Steady state photocarrier grating technique for diffusion length measurement in photoconductive insulators", Appl. Phys. Lett. 49, 791, 1986.

[2] R. Brüggemann in Advanced Characterization Techniques for Thin Film Solar Cells (Eds, D. Abou-Ras, T. Kirchartz, U. Rau), Wiley-VCH, Weinheim, Germany, 2011.

[3] C. Longeaud, "An automated steady state photocarrier grating experiment", Rev. Sci. Instrum 84, 055101, 2013.

[4] A. FathAllah, F. Ventosinos, C. Longeaud, "An automated experiment for determination of thin film semiconductor transport parameters", J. Phys. Conf. Ser. 558, 012011, 2014.

[5] A. Poruba, M. Vaněček, J. Rosa, L. Feitknecht, N. Wyrsch, J. Meier, A. Shah, T. Repmann, B. Rech, "Fourier transform photocurrent spectroscopy in thin film silicon solar cells", Proceedings of the $17^{\text {th }}$ European Photovoltaic Solar Energy Conference, 2981, 2001.

[6] M. Vaněček, A. Poruba, "Fourier-transform photocurrent spectroscopy of microcrystalline silicon for solar cells", Appl. Phys.Lett. 80, 719, 2002.

[7] C. Longeaud, F. Javier Ramos, A. Rebai, J. Rousset, "Impact of environmental stresses onto transport properties in hybrid perovskite investigated by steady state photocarrier grating and steady state photocurrent techniques", Sol. RRL, 1800192, 2018.

[8] J. F. Galisteo-Lopez, M. Anaya, M. E. Calvo, H. Miguez, "Environmental Effects on the Photophysics of Organic-Inorganic Halide Perovskites", J. Phys. Chem. Lett., 6, $2200,2015$.

[9] R. Brenes, C. Eames, V. Bulovic, M. Saiful Islam, S. D. Stranks, "The Impact of Atmosphere on the Local Luminescence Properties of Metal Halide Perovskite Grains", Adv. Mater., $1706208,2018$.

[10] A. Zohar, N. Kedem, I. Levine, D. Zohar, A. Vilan, D. Ehre, G. Hodes, D. Cahen, "Impedance Spectroscopic Indication for Solid State Electrochemical Reaction in (CH3NH3)PbI3 Films", J. Phys. Chem. Lett., 7, 191, 2016. 
